\title{
The strength of single-cell concrete box girders of deformable cross-section
}

\author{
R. J. S. SPENCE \& C. T. MORLEY
}

\section{Mr P. J. Cookson. Cambridge University}

The Authors have proposed a method for evaluating an upper bound on the collapse load of simply supported, single-cell box girders, where failure involved distortion of the cross-section. This mode of failure has been observed elsewhere, ${ }^{7}$ and it is apparent that for certain transverse reinforcement ratios it must be considered as an alternative to pure bending failure for eccentric loading.

45. However, in deriving the theoretical equation for the collapse locus, the Authors assume the concrete has infinite compressive strength. Finite compressive strength can be considered; the concrete is assumed to be rigid plastic with zero tensile strength, and the compressive strength is taken to be $-f_{\mathrm{o}} \mathrm{N} / \mathrm{nmm}^{2}$.

46. By way of illustration, a simple reinforcement distribution is assumed, but the equation is valid for any reinforcement symmetrical about the central vertical axis. The steel yields at $\pm f_{\mathrm{t}} \mathrm{N} / \mathrm{mm}^{2}$ and is uniformly distributed in the webs and fianges.

47. The mechanism is similar to that shown in Fig. 7, except that rotation of the heavily loaded web is about an axis $y^{\prime}$ below $\mathrm{A}$, and the top flange moves in its own plane, giving a midspan strain rate distribution as shown in Fig. 13(a) and stress distribution as in Fig. 13(b).

48. Using equilibrium to obtain the minimum upper bound, ${ }^{8}$ moments taken about the $y$ axis must be equal to zero for a simply supported beam. This is also true for the pure bending failure and hence $y^{\prime}$ must be the same in both cases. By considering the stress and strain rate diagrams it can be seen that the work done in the midspan hinge is half that for bending failure. Hence

$$
\frac{P}{2}+D=\frac{P_{\mathrm{b}}}{2}+\frac{4 M_{\mathrm{c}} L}{b} .
$$

where the twisting work in the diaphragms, webs and flanges has been neglected in accordance with $\$ 30$.

49. This equation may be compared with equation (18) written as

$$
\frac{P}{2}+D=\frac{P_{0}}{2}+\frac{4 M_{0} L}{b} \text {. . . . . . . . }
$$

The change is in the first term on the right. The difference between $P_{0}$ (the pure bending collapse load for infinite concrete strength) and $P_{\mathrm{b}}$ (the pure bending load for finite concrete strength) depends on the longitudinal reinforcement ratios, and the effect this has on equations (27) and (28) may be illustrated graphically. 
DISCUSSION

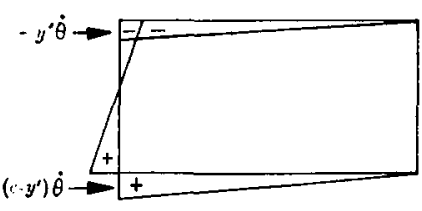

(a)

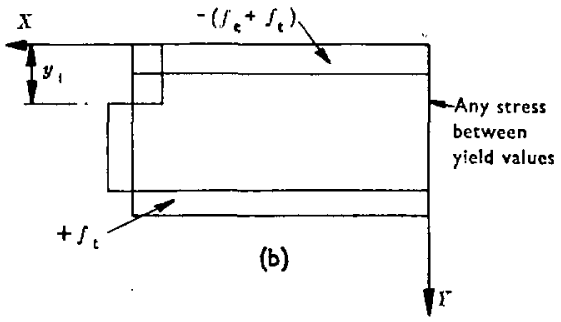

Fig. 13. Midspan strain rate and stress distributions

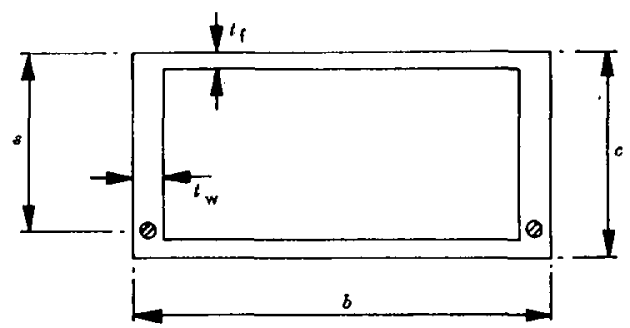

Fig. 14. Simplified beam cross-section

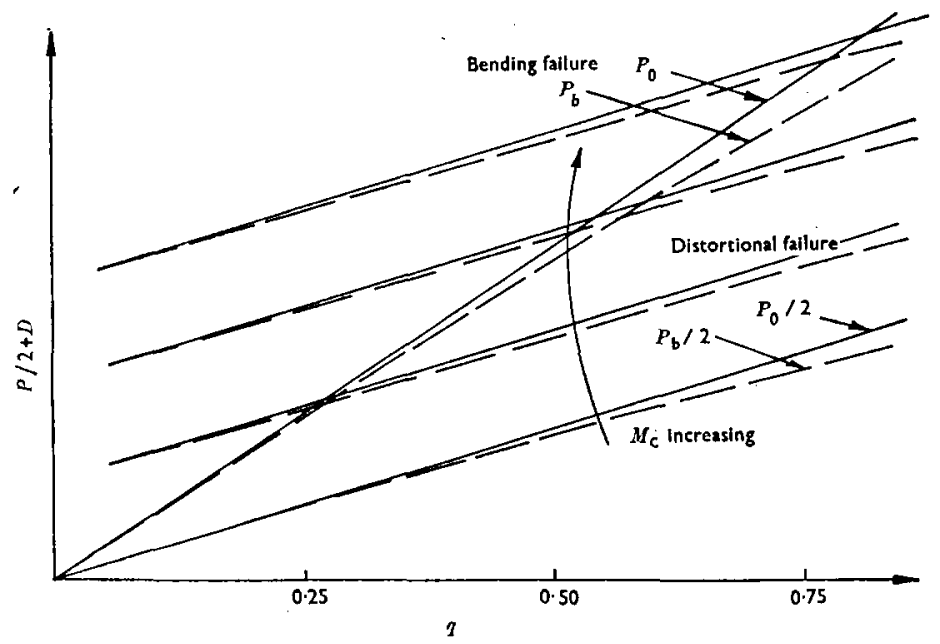

Fig. 15

476

Downloaded by [] on [26/04/23]. Copyright @ ICE Publishing, all rights reserved. 


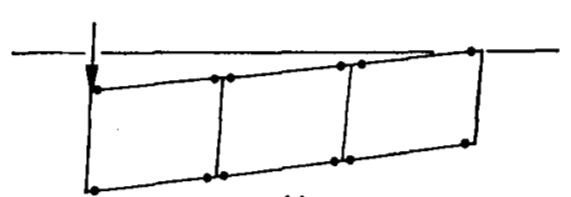

(a)

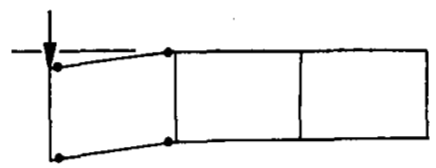

(b)

Fig. 16. Collapse modes for multi-cell beams

50. Consider the box shown in Fig. 14. Two concentrated areas of steel, each with a yield value of $F \mathrm{~N}$, lie at a depth $s \mathrm{~mm}$ below the top of the box. Dimensionless parameters are defined

It can be shown that

$$
\begin{gathered}
q=F / f_{\mathrm{c}} b t_{\mathrm{s}} \\
p=t_{\mathrm{f}} / t_{\mathrm{w}}
\end{gathered}
$$

$$
P_{\mathrm{b}}=P_{0}-g(q)
$$

where

$$
g(q)=8\left[\frac{\left(f_{\mathrm{c}}\right)\left(t_{\mathrm{f}}\right)^{2} b}{L}\right] q^{2}
$$

when the neutral axis lies in the top flange, i.e. $0 \leqslant q \leqslant \frac{1}{2}$, and

$$
g(q)=8\left[\frac{\left(f_{\mathrm{c}}\right)\left(f_{t}\right)^{2} b}{L}\right]\left[\frac{1 b}{8 t_{\mathrm{w}}}(2 q-1)^{2}+q-\frac{1}{4}\right]
$$

when the neutral axis lies in the webs, i.e. $\frac{1}{2} \leqslant q \leqslant \frac{1}{2}+\left(c-2 t_{t}\right) / p b$.

51. A graph may be drawn for a given size of box, and fixed value of $f_{\mathrm{c}}$, plotting failure load $(p / 2)+D$ against $q$, with parameter $M_{\mathrm{c}}$ (Fig. 15). Equation (28) is shown by unbroken lines. Equation (27) is shown by broken lines. The mechanism can be extended to simply supported multi-cell boxes (Fig. 16(a)) using the same arguments

$$
\frac{P}{2}+D=\frac{P_{\mathrm{b}}}{2}+\frac{n M_{\mathrm{c}} L}{b}
$$

where $n$ is the number of longitudinal hinges.

52. For larger values of $n$, pure bending failure is more probable, unless another mode of failure occurs, confined to one cell and involving shear (Fig. 16(b)). This type of mechanism is now being studied at Cambridge.

Professor V. Křistek, Department of Concrete Structures. Technical University. ĊVUT, Prague

Depending on the ratios of the dimensions of a girder's cross-section, its span and, in the case of a concrete structure, on the percentage and manner of reinforcement, box girders may be schematically divided into two groups according to whether the plastic hinges in the webs and flanges due to their plane action originate before or after the longitudinal plastic hinges in the cross-section corners. The first group includes girders whose transverse frame rigidity is maintained for long enough in the linearly elastic state. Box girders for bridges (particularly those made of concrete), however, are included in the second group. This means that the distortional character of the load produces the full transverse plastic moment $\bar{m}_{\mathrm{m}}$ in corners of the cross-section (Fig. 17(d)) far earlier than the full plastic moment $\bar{M}_{\mathrm{m}}$ in the webs. For this reason the plastic hinges originate first in the corners of that cross-section in which the deflexion due to distortion is greatest, i.e. where the transverse bending moment first attains the 
DISCUSSION

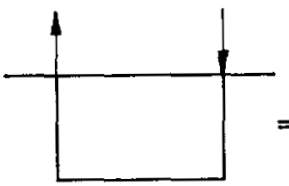

(a)

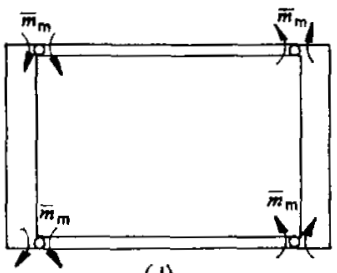

(d)

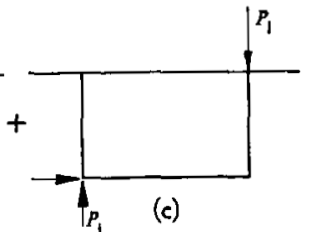

(b)

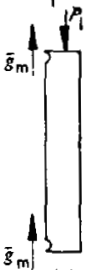

(e)

Fig. 17
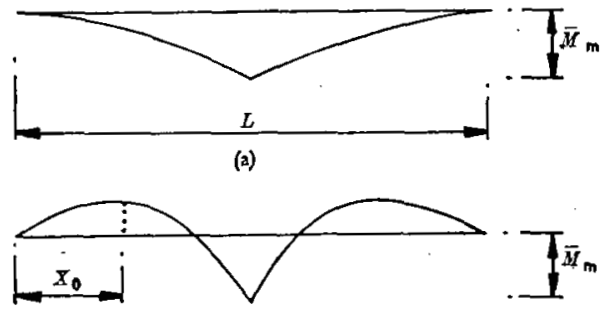

(b)

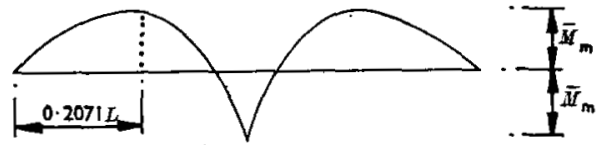

(c)

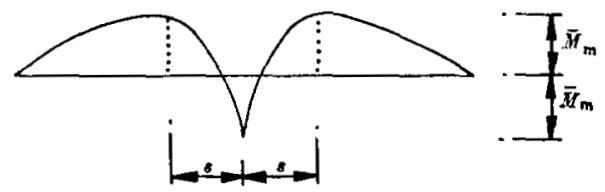

(d)

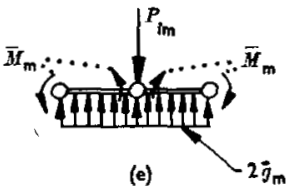

Fig. 18

478

Downloaded by [] on [26/04/23]. Copyright (C) ICE Publishing, all rights reserved. 
magnitude of $\bar{m}_{\mathrm{m}}$. With increasing deflexion these longitudinal hinges begin to expand along the girder edges. Afterwards the web in its own plane is stressed by the moment due to the difference of the vertical component of the external distortion load and the slab reactions $\bar{g}_{\mathrm{m}}$ corresponding to the full plastic moment of horizontal slabs $\bar{m}_{\mathrm{m}}$ under transverse frame behaviour of the cross-section. Their magnitude may be expressed by the relation

$$
\tilde{g}_{\mathrm{m}}=\frac{2 \bar{m}_{\mathrm{m}}}{b} \quad \text {. . . . . . . . . . }
$$

If the external distortional load is uniformly distributed, it has been proved that the longitudinal plastic hinges expand from the point of the greatest defiexion along the edges to almost the whole girder length. More complicated is the situation of loading by the local distortion effect (characterized by its vertical component $P_{1}$, Figs $17(\mathrm{c})$ and 17(e)). For box girders whose ultimate magnitudes of slab reactions $\bar{g}_{\mathrm{m}}$ (equation (29)) are inferior to $4 \bar{M}_{\mathrm{m}} / L^{2}$, the course of the loading process is similar to that for uniformly distributed load: the longitudinal plastic hinges expand gradually with increasing load, and the ultimate load is attained when the longitudinal bending moment in the web attains the value of $\bar{M}_{\mathrm{m}}$ at midspan, where a plastic hinge originates and, consequently, a mechanism with one degree of freedom is formed. For the ultimate value of the force $P_{1}$ it yields the relation

$$
P_{1 \mathrm{~m}}=\frac{4 \bar{M}_{\mathrm{m}}}{L}+\bar{g}_{\mathrm{m}} L \quad . \quad . \quad . \quad . \quad . \quad . \quad . \quad .
$$

The web bending moment diagram in this case has the form shown in Fig. 18(a).

54. If the values of the ultimate slab reactions of the box girder are within the limits of $4 \bar{M}_{\mathrm{m}} / L^{2}<\bar{g}_{\mathrm{m}}<23 \cdot 314 \bar{M}_{\mathrm{m}} / L^{2}$ the course of the loading process is similar; the ultimate value of the force $P_{1}$ is again determined by equation (30) and the plastic hinges in the web again originate at midspan, but the longitudinal bending moments in the end parts of the webs are negative (Fig. 18(b)); the highest negative bending moments originate in the cross-sections situated at a distance of

$$
x_{0}=\frac{L}{4}-\frac{\bar{M}_{\mathrm{m}}}{\bar{g}_{\mathrm{m}} L} \quad . \quad . \quad . \quad . \quad . \quad . \quad . \quad .
$$

from the supports, their magnitude being

$$
\bar{M}_{1}\left(x_{0}\right)=\frac{\bar{M}_{\mathrm{m}}}{2}-\frac{\bar{M}_{\mathrm{m}}^{2}}{\vec{g}_{\mathrm{m}} L^{2}}-\frac{\vec{g}_{\mathrm{m}} L^{3}}{16} \text {. . . . . . . }
$$

However, their absolute value is inferior to $\Pi_{\mathrm{m}}$.

55. The special case is characterized by the magnitude of the ultimate slab reaction $\bar{g}_{\mathrm{m}}=23.314 \bar{M}_{\mathrm{m}} / L^{2}$. In this case when the ultimate force attains the value of $P_{1}=$ $27 \cdot 314 \bar{M}_{\mathrm{m}} / L$, the full plastic moment $\bar{M}_{\mathrm{m}}$ is attained simultaneously in three crosssections, namely at midspan and in two cross-sections at a distance of $0.2071 L$ from the supports (Fig. 18(c)), where plastic hinges also originate.

56. In box girders characterized by the value of $\bar{g}_{\mathrm{m}}>23 \cdot 314 \bar{M}_{\mathrm{m}} / L^{2}$ the longitudinal plastic hinges can no longer extend as far as the supports, as the uniform upward load of the web $2 \bar{g}_{\mathrm{m}}$ (Fig. 17(e)) produced by them would effect web bending moments such that in combination with the effect due to the force $P_{1}$ at midspan it would be impossible for the full plastic moment $\overline{\boldsymbol{M}}_{\mathrm{m}}$ to be attained in any cross-section without producing a bending moment in another cross-section whose absolute value would exceed $\bar{M}_{\mathrm{m}}$. For $\bar{g}_{\mathrm{m}}=36 \bar{M}_{\mathrm{m}} / L^{2}$, for example, the force $P_{1}=48 \bar{M}_{\mathrm{m}} / L$ would produce at the point of $x_{0}=L / 6$ the bending moment of $\bar{M}_{1}(L / 6)=-\bar{M}_{\mathrm{m}}$, but at midspan the moment would be $\bar{M}_{1}(L / 2)=3 \bar{M}_{\mathrm{m}}$. The force $P_{1}=40 \bar{M}_{\mathrm{m}} / L$ would produce the bending moment $\bar{M}_{\mathrm{m}}$ at midspan, but in the cross-sections at distance of $x_{0}=0.222 \mathrm{~L}$ the moment of $\bar{M}_{1}$ $(0.222 L)=-1.778 \bar{M}_{\mathrm{m}}$ would be obtained. Thus the longitudinal plastic hinges in the edges of the box girder originate only in the central part of the span, where they produce the upward thrust $2 \bar{g}_{\mathrm{m}}$; in the end parts of the span the reactions due to the transverse 


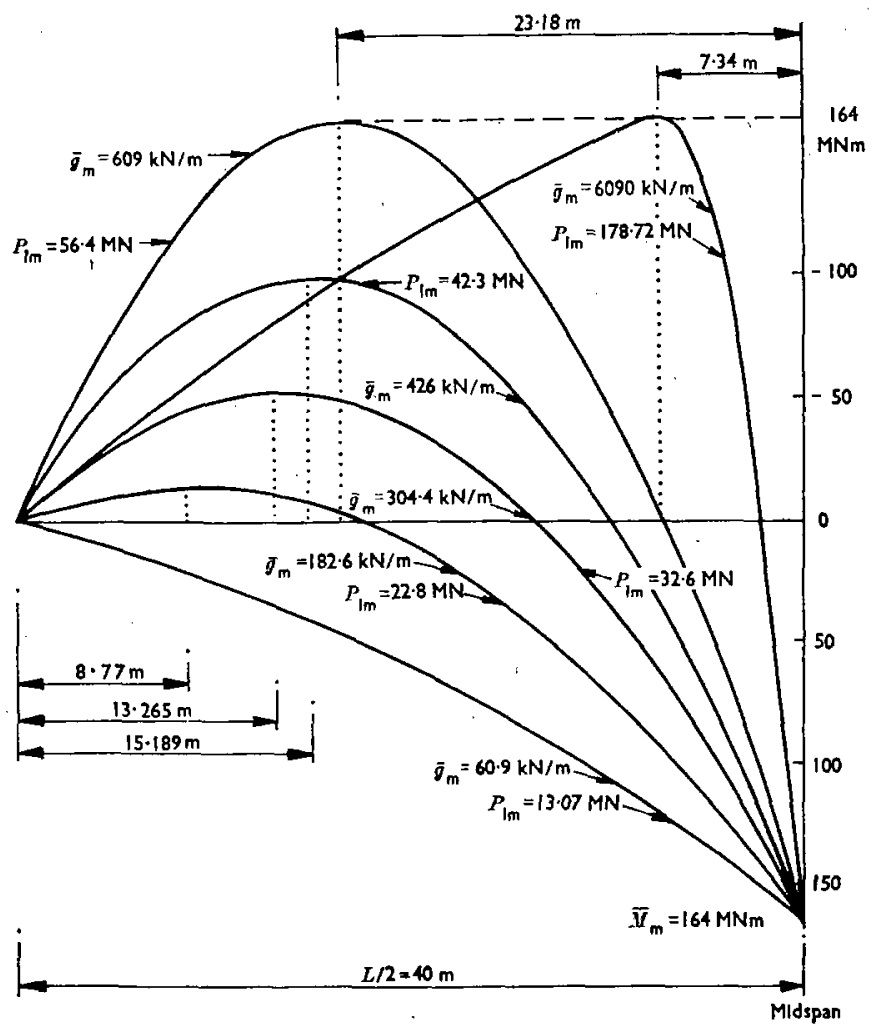

Fig. 19

frame rigidity of the box girder will be lower. Consequently, the diagram of the web moments due to these reactions will be straighter in these parts, thus, when added to the triangular moment diagram corresponding to the force applied at midspan, attaining the full plastic moment $\bar{M}_{\mathrm{m}}$ not only in the midspan cross-section, but also in another two cross-sections at a distance $s$ from the former (Fig. 18(d)). From the equilibrium of the mechanism between the hinges (Fig. 18(e)) it is possible to determine the ultimate value of the force $P_{1}$

$$
P_{1 \mathrm{~m}}=\frac{4}{s} \bar{M}_{\mathrm{m}}+2 \bar{g}_{\mathrm{m} s} \quad . \quad \cdot \quad \cdot \quad . \quad . \quad . \quad . \quad .
$$

57. The force $P_{1 \mathrm{~m}}$ attains its minimum value if

$$
s=\sqrt{ }\left(2 \bar{M}_{\mathrm{m}} / \bar{g}_{\mathrm{m}}\right)
$$

so that from equation (33) it holds that

$$
P_{1 \mathrm{~m}}=4 \sqrt{ }\left(2 \bar{M}_{\mathrm{m}} \bar{g}_{\mathrm{m}}\right)
$$

58. As all loads in the part of the box girder between the plastic hinges (Fig. 18(e)) are in equilibrium, i.e. $P_{1 \mathrm{~m}}-4 \bar{g}_{\mathrm{m}} s=0$, no reactions originate in the first and the third hinges, and the moment diagram has the form shown in Fig. 18(d). For the boundary case of $\bar{g}_{\mathrm{m}}=23.314 \bar{M}_{\mathrm{m}} / L^{2}$ equation (35) yields the values of $P_{1 \mathrm{~m}}=27.314 \bar{M}_{\mathrm{m}} / L$, thus confirming a connexion with the preceding procedure. 
59. The relations obtained were verified by a parametric study. The concrete box girder with a gradually increasing transverse frame rigidity was considered. The study covers a wide range of values of $\bar{g}_{\mathrm{m}}$, so that it includes the cases corresponding to all characteristic types of behaviour (Fig. 18). The first case has $\bar{g}_{\mathrm{m}}=60.9 \mathrm{kN} / \mathrm{m}$ which is inferior to $4 \bar{M}_{\mathrm{m}} / L^{2}=102.5 \mathrm{kN} / \mathrm{m}$; the bending moment diagram when the force $P_{1 \mathrm{~m}}$ has been attained has the form shown in Fig. 19 (cf. Fig. 18(a)); the moment diagrams of three other cases whose values of $\bar{g}_{\mathrm{m}}$ are in the range $102.5<\bar{g}_{\mathrm{m}}<23.314 \bar{M}_{\mathrm{m}} / L^{2}=$ $597 \mathrm{kN} / \mathrm{m}$ (Fig. 19) belong to the type shown in Fig. 18(b). The last two cases, of which particularly the second ranks among transversally highly resistant box girders in which the failure due to distortion load is difficult to imagine, characterized by the values of $\bar{g}_{\mathrm{m}}$ exceeding the boundary of $597 \mathrm{kN} / \mathrm{m}$, have moment diagrams (Fig. 19) whose form as well as the position of the zero points is in agreement with the typical form shown in Fig. 18(d).

60. In the Paper the applied point load is resolved into its symmetrical $P$ and antisymmetrical $D$ components (Fig. 6). The load $D$ denoted as 'distortional component of applied load' has two further components: the pure torsion (Fig. 17(b)) and the pure distortion (Fig. 17(c)). It seems to be reasonable to suppose that the pure distortional component is the decisive quantity for the distortional mode of failure. I think it may be expected that, if longitudinal plastic hinges arise in the corners of the cell, the corners can transfer a certain shear flow contributing to the twist which corresponds to the pure torsional component of the load applied.

\section{Dr Spence and Dr Morley}

Mr Cookson has proposed an interesting amendment to the assumed collapse mode, enabling proper theoretical allowance to be made for the limited compressive strength of concrete. In the Paper the device adopted was to assume infinite compressive strength in the theory, but' to compare theory with experiment by comparing the theoretical load ratio $P / P_{0}$ with the experimental ratio $P_{\mathrm{c}} / P_{\mathrm{b}}$. The test beams had low percentages of reinforcement, and the device adopted does not seem to have been seriously in error; the ratios of experimental collapse load to the theoretical collapse load for $\mathrm{Mr}$ Cookson's mechanism are always within 0.03 of the figures in the seventh column of Table 2.

62. Professor Kř́stek makes some interesting remarks, especially when he points out that hogging moments can occur in the loaded web near the supports, because of the forces acting between the flanges and the web. This effect can be especially important in concrete boxes, in which the hogging strength of the web may be markedly less than its strength against sagging moments. Recent work at Cambridge has shown that longitudinal shear forces between flange and web are of greater significance than shear forces normal to the flange planes. These longitudinal shear forces are not mentioned in the Paper because they do no work on the assumed collapse mechanism, but they have a decisive influence on the mechanism proposed by Mr Cookson in Fig. 16(b).

63. There is a need for care in decomposing applied loadings into components in situations involving plastic flow, for which the principle of superposition is not valid. For this reason the test loading cases were not decomposed further than into the symmetrical and anti-symmetrical components $P$ and $D$. This decomposition was thought to be illuminating, and is legitimate because the work done on the collapse mechanism is unaffected. Experimental work on box girders subjected to the purely local selfequilibrating load system in Fig. 17(c) would be very useful.

\section{References}

7. KRISTEK V. Influence of actual physical characteristics of material on the behaviour of box girders. Acta Tech., Praha, 1974, No. 5, 602-631.

8. Morley C. T. Yield line theory for reinforced concrete slabs at moderately large deflections. Mag. Concr. Res., 1967, 19, No. 61, 213. 